\title{
Recursive trigonometric interpolation algorithms
}

\author{
A A Stotsky \\ Computer Science and Engineering, Aalborg University at Esbjerg, Niels Bohrs Vej 8, Esbjerg 6700, Denmark. email: \\ stotskya@mail.ru
}

The manuscript was received on 3 June 2009 and was accepted after revision for publication on 2 October 2009.

DOI: 10.1243/09596518JSCE823

\begin{abstract}
Two new recursive algorithms for the calculation of the coefficients of the trigonometric polynomial that is fitted to measured data in the least-squares sense in a window of a sufficiently large size that is moving in time are proposed. Both algorithms use the strictly diagonally dominant property of the information matrix for a sufficiently large window size. The first one is based on a recursive inversion of the information matrix and the second one uses a priori estimate of the coefficients of the polynomial obtained from the Kaczmarz projection method. The algorithms are computationally suitable for selection of the order of a trigonometric polynomial in each step of a moving window in real-time applications. The results are verified by simulations.
\end{abstract}

Keywords: discrete Fourier transform, filtering, least squares, recursive inversion, signal processing, persistency of excitation

\section{INTRODUCTION}

The moving-in-time window discrete Fourier transform (DFT) method in which an oscillating signal is approximated via a trigonometric polynomial in a moving window of a certain size is widely used for both the estimation of the frequency contents of the signals and filtering in real-time signal processing applications. The orthogonality property of the trigonometric polynomials applied in a DFT method implies a restriction on the size of the moving window. The choice of the window size has a direct impact on the variance of the estimated parameters and on the estimated variance of the measurement noise which is calculated as the sum of the squared approximation errors divided by the number of degrees of freedom (the window size minus a number of unknown parameters). The restrictions applied to the window size in the DFT method might imply a poor estimation performance of both unknown parameters and variance of the measurement noise. Fortunately, a periodic signal can be approximated via a trigonometric polynomial in a least-squares sense for any window size for estimation of the frequency contents of the signal. This method is called a moving-in-time window trigonometric interpolation (TI) method and requires a computationally expensive inversion of an information matrix.

In this paper trigonometric interpolation is understood to be the process of approximation of given data points via a trigonometric polynomial (a sum of sines and cosines of given frequencies) in the leastsquares sense. The size of the moving window (the number of measured data points) is larger than the number of unknown coefficients of the polynomial.

A recursive algorithm based on a matrix inversion lemma for a matrix inversion that reduces the computational burden for the TI method accumulates errors in a finite precision implementation environment [1]. Moreover, new frequencies can appear in the oscillating signal during a system operation. The order of the trigonometric polynomial should be selected in each step of a moving window in this case. In this paper the number of frequencies is taken to be the order of the trigonometric polynomial. The order of the polynomial should be increased (new frequencies should be added to a model) until the estimate of the variance of the measurement noise calculated from the approximation error is reduced and this reduction is statistically significant. The TI method becomes very computationally expensive especially for the case of a large number of frequencies since it 
requires the inversion of the information matrix in each step of the moving window [2]. This paper describes two new computationally efficient algorithms for calculation of the coefficients of the trigonometric polynomial in the TI method.

The information matrix, which is inverted in order to estimate the coefficients of the polynomial, is defined as the sum of rank one matrices calculated via a multiplication of the regressor column vector by its transpose in each step of the moving-in-time window. The regressor vector consists of trigonometric functions (sines and cosines) at different frequencies. The diagonal of the information matrix includes the sum of squares of the trigonometric functions which can be decomposed into two parts: the average part that is proportional to the window size and the periodic part. All other elements of the information matrix only have a periodic part. Therefore, this matrix can be made a strictly diagonally dominant (SDD) matrix for a sufficiently large window size. A matrix is said to be SDD if in every row of the matrix, the magnitude of the diagonal entry in that row is larger than the sum of the magnitudes of all the other (non-diagonal) entries in that row. By the Gershgorin circle theorem, an SDD matrix is non-singular, and if the SDD matrix is symmetric and all main diagonal entries are positive then all the eigenvalues of this matrix are real and positive (this result is also known as the LevyDesplanques theorem [3]). The dominant part of the SDD matrix is its diagonal. An inverse of the diagonal matrix whose diagonal when taken from the SDD matrix is close to the SDD matrix inverse can be used as a starting point for a recursive calculation of the inverse of the information matrix. In other words this property allows:

(a) the design of the diagonal matrix as an approximate of the information matrix inverse;

(b) proving that the regressor is persistently exciting which is an important property for the parameter convergence in estimation algorithms.

The main contributions of this paper are based on the diagonal dominance property of the information matrix and can be summarized as follows:

(a) a new algorithm for recursive inversion of the information matrix;

(b) a comparison of the convergence rate for the algorithm mentioned above with the convergence rate of known algorithms;

(c) a new algorithm for a direct calculation of the parameters with a priory estimates obtained from the Kaczmarz projection method.
Both proposed algorithms use priory estimates which are available due to the SDD property of the information matrix, and improve these estimates recursively.

\section{PROBLEM STATEMENT AND PROPERTIES OF THE INFORMATION MATRIX}

\subsection{Problem statement}

Suppose that a measured oscillating signal can be presented in the following form

$$
y_{k}=\boldsymbol{\varphi}_{k}^{\mathrm{T}} \boldsymbol{\theta}_{*}+\xi_{k}
$$

where $\varphi_{k}$ is the regressor and $\boldsymbol{\theta}_{*}$ is the vector of constant unknown parameters defined as follows

$$
\begin{aligned}
& \boldsymbol{\varphi}_{k}^{\mathrm{T}}= {[1 \cos (k \Delta) \sin (k \Delta) \cos (2 k \Delta)} \\
&\sin (2 k \Delta) \ldots \cos (n k \Delta) \sin (n k \Delta)] \\
& \boldsymbol{\theta}_{*}^{\mathrm{T}}=\left[\theta_{0 *} \theta_{1 *} \theta_{2 *} \theta_{3 *} \theta_{4 *} \ldots \theta_{(2 n-1) *} \theta_{(2 n) *}\right]
\end{aligned}
$$

where $k=1,2, \ldots$ is the step number, $\Delta>0$ is the step size, $\xi_{k}$ is a zero mean white Gaussian noise with a variance $\sigma^{2}$. The measured periodic signal is presented as a trigonometric polynomial with known frequencies and unknown coefficients, whereas the regressor (2) includes known trigonometric functions $\cos (k q \Delta) \sin (k q \Delta)$, where $q=1,2, \ldots, n$ is the frequency, $n$ is the number of frequencies involved and the order of the trigonometric polynomial, if at least one of the coefficients $\theta_{(2 n-1) *}$ or $\theta_{(2 n) *}$ is not equal to zero.

The model of the signal (1) is presented in the following form

$$
\hat{y}_{k}=\boldsymbol{\varphi}_{k}^{\mathrm{T}} \boldsymbol{\theta}_{k}
$$

with adjustable parameters

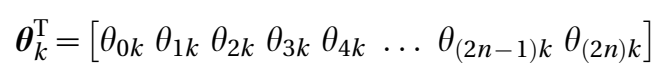

Introducing a moving window of size $w$ and an error

$$
E_{k}=\sum_{j=k-(w-1)}^{j=k}\left(y_{j}-\hat{y}_{j}\right)^{2}, \text { for } k=w, w+1, \ldots
$$

a least-squares solution that minimizes the performance index (6) is as follows 


$$
\boldsymbol{\theta}_{k}=\left[\sum_{j=k-(w-1)}^{j=k} \boldsymbol{\varphi}_{j} \boldsymbol{\varphi}_{j}^{\mathrm{T}}\right]^{-1} \sum_{j=k-(w-1)}^{j=k} \boldsymbol{\varphi}_{j} y_{j}
$$

where the matrix $\sum_{j=k-(w-1)}^{j=k} \boldsymbol{\varphi}_{j} \boldsymbol{\varphi}_{j}^{\mathrm{T}}$ is often called the information matrix. In order to calculate the parameter vector $\boldsymbol{\theta}_{k}$ this information matrix should be inverted in each step $k$ of the moving window. This is a computationally expensive procedure, if the size of the parameter vector is large. This matrix has a remarkable property that helps in reduction of the computational burden when calculating the parameter vector for a sufficiently large window size. This property is described in the section 2.2.

\subsection{Properties of the information matrix}

\subsubsection{A key property of the information matrix}

\section{Lemma 1}

There exists a window size $w_{*}$ such that for all

$$
w>w_{*}
$$

where $w_{*}$ is defined in equation (60), the following matrix

$$
\mathbf{A}=\sum_{j=k-(w-1)}^{j=k} \boldsymbol{\varphi}_{j} \boldsymbol{\varphi}_{j}^{\mathrm{T}}
$$

where $k=w, w+1, w+2, w+3, \ldots$, and

$$
\boldsymbol{\varphi}_{j}^{\mathrm{T}}=[1 \cos (j \Delta) \sin (j \Delta) \ldots \cos (j n \Delta) \sin (j n \Delta)]
$$

is an SDD matrix, i.e. the following inequality holds

$$
\left|a_{i i}\right|>\sum_{j=1, j \neq i}^{2 n+1}\left|a_{i j}\right|, \text { for } i=1, \ldots,(2 n+1)
$$

Moreover, the following inequality is also true

$$
0<\alpha \mathbf{I} \leqslant \sum_{j=k-(w-1)}^{j=k} \boldsymbol{\varphi}_{j} \boldsymbol{\varphi}_{j}^{\mathrm{T}} \leqslant \beta \mathbf{I}
$$

where $\alpha>0$ and $\beta>0$ are minimal and maximal eigenvalues of the matrix $\mathbf{A}$ respectively.

Lemma 1 is proved via a straightforward evaluation of the elements of matrix (9). This evaluation is performed in Appendix 1. Some of the non-diagonal and diagonal elements are listed below for the sake of clarity

$$
\begin{aligned}
& \sum_{j=k-(w-1)}^{j=k} \cos (j q \Delta) \\
& =\frac{\sin (w q \Delta / 2) \cos [(2 k+1-w) / 2 q \Delta]}{\sin (q \Delta / 2)} \\
& \sum_{j=k-(w-1)}^{j=k} \sin (j q \Delta) \\
& =\frac{\sin (w q \Delta / 2) \sin [(2 k+1-w) / 2 q \Delta]}{\sin (q \Delta / 2)}
\end{aligned}
$$

$$
\begin{aligned}
& \sum_{j=k-(w-1)}^{j=k} \sin ^{2}(j q \Delta)=\frac{w}{2} \\
& -\frac{\sin (w q \Delta) \cos [(2 k+1-w) q \Delta]}{2 \sin (q \Delta)}
\end{aligned}
$$

$$
\begin{gathered}
\sum_{j=k-(w-1)}^{j=k} \cos ^{2}(j q \Delta)=\underbrace{\frac{w}{2}}_{\text {average part }} \\
+\underbrace{\frac{\sin (w q \Delta) \cos [(2 k+1-w) q \Delta]}{2 \sin (q \Delta)}}_{\text {periodic part }}
\end{gathered}
$$

where $q=1,2, \ldots, n$. Notice that diagonal elements of the matrix A defined by equations (15) and (16) have an average part which is proportional to the window size $w$ while all other elements of this matrix have a periodic part only. Enlarging the window size $w$ gives the diagonal dominance.

\section{Remark 1}

In the following a sufficiently large window size is understood to be a window size that satisfies inequality (8). This sufficiently large window size might not be large in absolute values.

\section{Corollary 1}

The matrix $\mathbf{A}$ is a diagonal matrix if $\Delta=2 \pi / w$. Indeed, a set of functions $\{\cos (j q \Delta), \sin (j q \Delta)\}, q=1$, $2, \ldots, n, j=1, \ldots, w$ is orthogonal in the interval $j \Delta=[\Delta, 2 \Delta, 3 \Delta, \ldots, w \Delta]=[\Delta, 2 \Delta, 3 \Delta, \ldots, 2 \pi]$. This implies that $\sin (w q \Delta)=\sin (2 \pi q)=0$, and $\sin (w q \Delta /$ $2)=\sin (\pi q)=0$. Therefore, the periodic parts in equations (15) and (16) are equal to zero as well as the periodic parts in equations (13) and (14) provided that $q / 2$ and $q$ are not a multiple of $w$ so 
that $\sin (q \Delta) \neq 0$ and $\sin (q \Delta / 2) \neq 0$. The matrix $\mathbf{A}$ defined as

$$
\mathbf{A}=\operatorname{diag}\left(w \frac{w}{2} \ldots \frac{w}{2}\right)
$$

can be easily inverted in this case and therefore $\boldsymbol{\theta}_{k}$ defined in equation (7) can be written as follows

$$
\begin{aligned}
& \theta_{0 k}=\frac{1}{w} \sum_{j=k-(w-1)}^{j=k} y_{j} \\
& \theta_{1 k}=\frac{2}{w} \sum_{j=k-(w-1)}^{j=k} y_{j} \cos (j \Delta) \\
& \theta_{2 k}=\frac{2}{w} \sum_{j=k-(w-1)}^{j=k} y_{j} \sin (j \Delta) \\
& \ldots \quad \ldots y_{(2 n) k}=\frac{2}{w} \sum_{j=k-(w-1)}^{j=k} y_{j} \sin (j n \Delta)
\end{aligned}
$$

The coefficients (17) to (20) are called the Fourier coefficients that are applied in a DFT method. The key advantage of the DFT method is a parameter decomposition with respect to frequencies due to the diagonal information matrix. This allows the design of a filter with a low computational complexity at a single frequency. The choice of the window size $w$ that should satisfy the orthogonality condition is the main restriction to the improvement of the accuracy of estimation of both the variance of the measurement noise

$$
\hat{\sigma}^{2}=\frac{E_{k}}{w-2 n-1}
$$

and parameter vector $\boldsymbol{\theta}_{k}$. Accuracy of the estimate of the parameter vector and the variance of the measurement noise is improved via increasing the window size [4]. Notice that a large window size might have a negative impact on the transient performance of the estimation.

\section{Corollary 2}

The inequality (12) can be written in the following form after a simple change of variables

$$
0<\alpha \mathbf{I} \leqslant \sum_{j=k}^{j=k+w} \boldsymbol{\varphi}_{j} \boldsymbol{\varphi}_{j}^{\mathrm{T}} \leqslant \beta \mathbf{I}, \quad \text { for } \quad k=1,2, \ldots
$$

with the regressor $\boldsymbol{\varphi}_{j}$ being defined in equation (12). It can be seen as a property of a persistency of excitation of the regressor which shows that there exist positive constants $\alpha$ and $\beta$ for a sufficiently large window size $w$ such that inequalities (21) holds. The basis of the property of the persistency of excitation is a strictly diagonally dominance of the matrix $\sum_{j=k}^{j=k+w} \boldsymbol{\varphi}_{j} \boldsymbol{\varphi}_{j}^{\mathrm{T}}$ for a sufficiently large $w$. The property of a persistency of excitation established for trigonometric polynomials is an important property for a parameter convergence in the system identification and adaptive control methods.

\subsubsection{A property of the diagonal of the information matrix}

Suppose that the window size $w$ is large enough so that the matrix A defined in equation (9) is an SDD matrix. Introduce the following matrix

$$
\mathbf{D}=\operatorname{diag}\left(a_{11}, a_{22}, \ldots, a_{m m}\right)
$$

where $a_{11}, a_{22}, \ldots$ are diagonal elements of the matrix A defined in equations (15) and (16). This matrix $\mathbf{D}$ is invertible, and its inverse can be used as an approximation of $\mathbf{A}^{-1}$. All the diagonal elements of the matrix $\mathbf{D}^{-1} \mathbf{A}$ are equal to one. All the diagonal elements of the matrix $\mathbf{I}-\mathbf{D}^{-1} \mathbf{A}$ are equal to zero and there exists a positive number $\kappa$ such that the following is true

$$
\left\|\mathbf{I}-\mathbf{D}^{-1} \mathbf{A}\right\|_{\infty} \leqslant \kappa<1
$$

where $\mathbf{A}$ is an SDD matrix, and the norm $\|\cdot\|_{\infty}$ is the maximum row sum matrix norm. This key property of the diagonal matrix $\mathbf{D}$ is the basis for two recursive algorithms described in section 3 .

\section{RECURSIVE INVERSION OF THE INFORMATION MATRIX}

A recursive calculation of the inverse of $\mathbf{A}$ is based on the Lemma 2.

Lemma 2

The inverse of an $n \times n$ matrix $\mathbf{C}$ can be calculated as follows 


$$
\mathbf{C}^{-1}=\sum_{i=0}^{\infty}(\mathbf{I}-\mathbf{C})^{i}
$$

provided that equation (23) holds with $\mathbf{C} \stackrel{\text { def }}{=} \mathbf{D}^{-1} \mathbf{A}$.

The proof is presented in Appendix 2.

If the approximate inverse of $\mathbf{A}$ is known via $\mathbf{D}^{-1} \approx \mathbf{A}^{-1}$ and inequality (23) holds then the following recursive algorithm for calculation of $\mathbf{A}^{-1}$ is proposed

$$
\begin{aligned}
& \mathbf{F}_{1}=\mathbf{I}, \quad \mathbf{G}_{1}=\left(\mathbf{I}-\mathbf{D}^{-1} \mathbf{A}\right) \\
& \mathbf{G}_{i}=\mathbf{G}_{i-1} \mathbf{G}_{1} \\
& \mathbf{F}_{i}=\mathbf{F}_{i-1}+\mathbf{G}_{i-1}
\end{aligned}
$$

where $i=2,3, \ldots$ The algorithm is stopped if $\left\|\mathbf{I}-\mathbf{D}^{-1} \mathbf{A F}_{i}\right\| \leqslant \varepsilon$, where $\varepsilon$ is a small positive number.

Algorithm (27) gives a recursive estimate of $\mathbf{A}^{-1}$, namely $\mathbf{D}^{-1} \mathbf{A F}_{i} \rightarrow \mathbf{I}$ as $i \rightarrow \infty$. This implies that $\mathbf{F}_{i} \mathbf{D}^{-1} \rightarrow \mathbf{A}^{-1}$ as $i \rightarrow \infty$. Indeed, algorithm (27) can be written in the following form

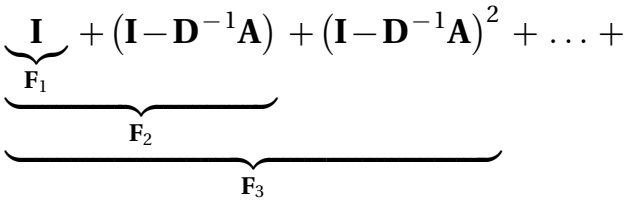

$$
\begin{aligned}
& =\sum_{i=0}^{N}\left(\mathbf{I}-\mathbf{D}^{-1} \mathbf{A}\right)^{i}
\end{aligned}
$$

The sum $\sum_{i=0}^{N}\left(\mathbf{I}-\mathbf{D}^{-1} \mathbf{A}\right)^{i} \rightarrow\left(\mathbf{D}^{-1} \mathbf{A}\right)^{-1}$ as $N \rightarrow \infty$ due to Lemma 2 with $\mathbf{C}=\left(\mathbf{D}^{-1} \mathbf{A}\right)$ since inequality (23) is valid and

$$
\underbrace{\left(\sum_{i=0}^{N}\left(\mathbf{I}-\mathbf{D}^{-1} \mathbf{A}\right)^{i}\right)}_{F_{i}} \mathbf{D}^{-1} \rightarrow \mathbf{A}^{-1} \text { as } N \rightarrow \infty
$$

The convergence rate of the algorithm is determined by upper-bound $\kappa$ of the $\left\|\mathbf{I}-\mathbf{D}^{-1} \mathbf{A}\right\|$ and is relatively fast for $\kappa \ll 1$. This convergence rate is evaluated in Appendix 3. The parameter vector $\boldsymbol{\theta}_{k}$ defined in equation (7) can easily be calculated as soon as the information matrix is inverted.

\section{Remark 2}

The following algorithm (see [2] and references therein) can also be applied for calculation of the inverse of matrix $\mathbf{A}$

$$
\begin{aligned}
& \mathbf{G}_{i}=\mathbf{G}_{i-1}+\mathbf{F}_{i-1} \mathbf{G}_{i-1} \\
& \mathbf{F}_{i}=\mathbf{I}-\mathbf{G}_{i} \mathbf{A} \\
& \mathbf{F}_{0}=\mathbf{I}-\mathbf{G}_{0} \mathbf{A} \\
& \mathbf{G}_{0}=\mathbf{D}^{-1} \\
& \left\|\mathbf{F}_{0}\right\| \stackrel{(23)}{\leqslant} \kappa<1
\end{aligned}
$$

where $i=1,2,3, \ldots$ and $\lim _{i \rightarrow \infty} \mathbf{G}_{i}=\mathbf{A}^{-1}$. A convergence rate of the algorithm (30) to (34) is evaluated in Appendix 3. A comparative analysis of the convergence rate of algorithms (30) to (34) and (25) to (27) is also performed in Appendix 3.

\section{Remark 3}

The third algorithm for the recursive calculation of the inverse of matrix $\mathbf{A}$ is a Newton-type algorithm which can be described as follows

$$
\begin{aligned}
& \mathbf{U}_{i}=\left[\sum_{j=0}^{N} \boldsymbol{\Psi}_{i-1}^{j}\right] \mathbf{U}_{i-1} \\
& \boldsymbol{\Psi}_{i}=\mathbf{I}-\mathbf{U}_{i} \mathbf{A} \\
& \mathbf{U}_{0}=\mathbf{D}^{-1} \\
& \boldsymbol{\Psi}_{0}=\mathbf{I}-\mathbf{D}^{-1} \mathbf{A} \\
& \left\|\boldsymbol{\Psi}_{0}\right\| \stackrel{(23)}{\leqslant} \kappa<1
\end{aligned}
$$

where $N$ is the algorithm parameter, $i=1,2,3, \ldots$ and $\lim _{i \rightarrow \infty} \mathbf{U}_{i}=\mathbf{A}^{-1}$. Indeed, $\sum_{j=0}^{N} \boldsymbol{\Psi}_{i-1}^{j} \rightarrow\left(\mathbf{I}-\boldsymbol{\Psi}_{i-1}\right)^{-1}$ as $N \rightarrow \infty$ (see equation (62)) provided that $\left\|\boldsymbol{\Psi}_{i-1}\right\|<1$, and hence

$$
\begin{gathered}
\mathbf{U}_{i}=\left[\sum_{j=0}^{N} \boldsymbol{\Psi}_{i-1}^{j}\right] \mathbf{U}_{i-1} \rightarrow\left(\mathbf{I}-\boldsymbol{\Psi}_{i-1}\right)^{-1} \mathbf{U}_{i-1} \\
\stackrel{(36)}{=}\left(\mathbf{U}_{i-1} \mathbf{A}\right)^{-1} \mathbf{U}_{i-1}=\mathbf{A}^{-1} \quad \text { as } \quad N \rightarrow \infty .
\end{gathered}
$$


The algorithm parameter $N$ that determines the number of terms in the sum $\sum_{j=0}^{N} \boldsymbol{\Psi}_{i-1}^{j}$ is a finite number. The error $\mathbf{U}_{i}-\mathbf{A}^{-1}$ is successively reduced and the sequence of matrices $\mathbf{U}_{i}$ converges to $\mathbf{A}^{-1}$ as $i=1,2$, $3, \ldots$ and $\left\|\boldsymbol{\Psi}_{i}\right\|$ converges to zero. A convergence rate of algorithm (35) to (39) can be evaluated similar to evaluation of the convergence rate of algorithms (30) to (34) and (25) to (27) described in Appendix 3.

The TI algorithm described above can be seen as an extension of the DFT method. Indeed, each element of the diagonal matrix $\mathbf{D}$ defined in equation (22) drawn from the information matrix in the TI method has both an average part and a periodic part. The periodic part is equal to zero in the DFT method and a diagonal matrix whose elements consist only of the average part is inverted in order to calculate the parameter vector. Each element of the inverse of the diagonal matrix $\mathbf{D}$ that contains the most valuable (dominant) part of the information matrix, has both an average part and a periodic part in the TI method. This inverse is a starting point for recursive estimation of the inverse of information matrix $\mathbf{A}$, where the convergence is guaranteed by the inequality (23). Periodic parts are added to the average parts in the diagonal matrix in the TI method that allows a calculation of the inverse of the information matrix in the case where orthogonality is not valid.

Application of this algorithm is restricted mainly to the case where the size of the parameter vector $\boldsymbol{\theta}_{k}$ is low, and a window size is large enough so that $\kappa \ll 1$. This algorithm is computationally expensive if the size of the parameter vector is large and $\kappa$ is close to one. The second algorithm which is applicable in this case is described in section 4 . This algorithm has two parts: the first part is a preliminary estimation of the parameter vector by means of the Kaczmarz algorithm using the property of persistency of excitation, see equation (21), and the second part is a recursive improvement of the estimates. Recursive matrix inversion is avoided in this algorithm and preliminary estimates are provided by the Kaczmarz algorithm. Since the Kaczmarz algorithm provides estimates that are relatively close to the parameter vector $\boldsymbol{\theta}_{k}$ a recursive algorithm just takes a couple of steps in order to achieve a desired accuracy of the parameter estimation. Notice that the Kaczmarz algorithm cannot be applied alone in some cases since it neither allows the reliable selection of the order of a trigonometric polynomial in the case where new frequencies appear in the signal nor estimation of the variance of measurement noise. Therefore, it can just be used as a tool to get a preliminary estimate of the vector $\boldsymbol{\theta}_{k}$.

\section{PARAMETER ESTIMATION WITH THE KACZMARZ ALGORITHM AND RECURSIVE CALCULATION OF THE PARAMETERS}

\subsection{The Kaczmarz projection method}

Suppose that the signal is described by equation (1) and the model of the signal is given by $\hat{y}_{k}=\varphi_{k}^{\mathrm{T}} \boldsymbol{\vartheta}_{k}$. Consider the following Kaczmarz update algorithm for a vector $\vartheta_{k}$

$$
\boldsymbol{\vartheta}_{k}=\vartheta_{k-1}+\frac{\boldsymbol{\varphi}_{k}}{n+1}\left(y_{k}-\boldsymbol{\varphi}_{k}^{\mathrm{T}} \boldsymbol{\vartheta}_{k-1}\right)
$$

Straightforward calculations show that $\tilde{\boldsymbol{\vartheta}}_{k}^{\mathrm{T}} \boldsymbol{\varphi}_{k}=\xi_{k}$, where $\tilde{\boldsymbol{\vartheta}}=\boldsymbol{\vartheta}_{k}-\boldsymbol{\theta}_{*}$. Multiplying this identity by $\boldsymbol{\varphi}_{k}^{\mathrm{T}}$ and taking the sum yields $\sum_{k=r}^{r+w} \tilde{\boldsymbol{\vartheta}}_{k}^{\mathrm{T}}\left(\boldsymbol{\varphi}_{k} \boldsymbol{\varphi}_{k}^{\mathrm{T}}\right)=\sum_{k=r}^{r+w} \boldsymbol{\varphi}_{k}^{\mathrm{T}} \xi_{k}, r=1,2$, ... and the following mathematical expectation is equal to zero

$$
E\left[\sum_{k=r}^{r+w} \tilde{\boldsymbol{\vartheta}}_{k}^{\mathrm{T}}\left(\boldsymbol{\varphi}_{k} \boldsymbol{\varphi}_{k}^{\mathrm{T}}\right)\right]=\sum_{k=r}^{r+w} \boldsymbol{\varphi}_{k}^{\mathrm{T}} E\left[\xi_{k}\right]=0,
$$

since $\xi_{k}$ is a zero mean noise. Notice that equation (40) represents a low pass filter driven by the oscillating input $\varphi_{k} y_{k} /(n+1)$. Therefore, the parameter vector $\vartheta_{k}$ changes slower than the input $\varphi_{k} y_{k} /(n+1)$ and a mathematical expectation of the vector of the adjustable parameters $\vartheta_{k}$ tends to become an approximately constant (slowly varying) vector after some transients. Therefore, $E\left[\tilde{\boldsymbol{\theta}}_{k}^{\mathrm{T}}\right]$ can be taken out of the sum for a sufficiently large $k$ which in turn implies that

$$
E\left[\tilde{\boldsymbol{\vartheta}}_{k}^{\mathrm{T}}\right]\left[\sum_{k=r}^{r+w} \boldsymbol{\varphi}_{k} \boldsymbol{\varphi}_{k}^{\mathrm{T}}\right]=0
$$

and the estimate $\vartheta_{k}$ of $\boldsymbol{\theta}_{*}$ given by equation (40) is consequentlyunbiased,i.e. $E\left[\tilde{\boldsymbol{\theta}}_{k}^{\mathrm{T}}\right]=0$ since the condition of the persistency of excitation (21) is valid for trigonometric polynomials.

\subsection{Recursive calculation of the parameters}

Suppose that the estimate $\vartheta_{k}$ of the vector of true parameters $\boldsymbol{\theta}_{*}$ is calculated in each step $k$ using Kaczmarz algorithm (40). The least-squares estimate $\boldsymbol{\theta}_{k}$ (in each step $k$ ) defined in equation (7) can recursively be calculated for all the window sizes which satisfy equation (8) as follows

$$
\boldsymbol{\theta}_{i}=\boldsymbol{\theta}_{i-1}-\mathbf{D}^{-1}\left(\mathbf{A} \boldsymbol{\theta}_{i-1}-\boldsymbol{b}\right), \quad \boldsymbol{\theta}_{0}=\boldsymbol{\vartheta}_{k}
$$

where matrix $\mathbf{D}$ is defined in equation (22), matrix $\mathbf{A}$ 
in equation (9) and vector $\boldsymbol{b}$ is defined as follows $\boldsymbol{b}=\sum_{j=k-(w-1)}^{j=k} \boldsymbol{\varphi}_{j} y_{j}, \quad i=1,2, \ldots, \boldsymbol{\theta}_{k}=\mathbf{A}^{-1} \boldsymbol{b}$ due to equation (7) and $\vartheta_{k}$ is defined in equation (40). Straightforward calculations show that the following error equation is valid $\boldsymbol{x}_{i}=\left(\mathbf{I}-\mathbf{D}^{-1} \mathbf{A}\right) \boldsymbol{x}_{i-1}$, where $\boldsymbol{x}_{i}=\boldsymbol{\theta}_{i}-\boldsymbol{\theta}_{k}$. This difference equation has the following solution: $\boldsymbol{x}_{i}=\left(\mathbf{I}-\mathbf{D}^{-1} \mathbf{A}\right)^{i} \boldsymbol{x}_{0}$, where $\boldsymbol{x}_{0}=\boldsymbol{\vartheta}_{k}-\boldsymbol{\theta}_{k}$, and inequality (23) is true since matrix $\mathbf{A}$ is an SDD matrix. Evaluation of the upper bound of the norm of the error yields

$$
\begin{aligned}
& \left\|x_{i}\right\| \\
& \stackrel{(23)}{\leqslant}\left\|\left(\mathbf{I}-\mathbf{D}^{-1} \mathbf{A}\right)^{i}\right\|\left\|\mathbf{x}_{0}\right\| \leqslant\left\|\left(\mathbf{I}-\mathbf{D}^{-1} \mathbf{A}\right)\right\|^{i}\left\|\mathbf{x}_{0}\right\| \\
& \stackrel{\kappa^{i}}{ }\left\|\mathbf{x}_{0}\right\|
\end{aligned}
$$

Since $\vartheta_{k} \approx \theta_{k}$ the following norm $\left\|\mathbf{x}_{0}\right\|$ is a sufficiently small number and few steps only are required to achieve the desired accuracy of the error $\boldsymbol{x}_{i}$.

\section{SIMULATION RESULTS}

Consider a problem of reconstruction of the first harmonic of a periodic signal which is described by a third-order trigonometric polynomial with noisy measurements

$$
\begin{aligned}
& y_{k}=\boldsymbol{\varphi}_{k}^{\mathrm{T}} \boldsymbol{\theta}_{*}+\xi_{k} \\
& \boldsymbol{\varphi}_{k}^{\mathrm{T}}=[1 \cos (k \Delta) \sin (k \Delta) \cos (2 k \Delta) \\
& \sin (2 k \Delta) \cos (3 k \Delta) \sin (3 k \Delta)] \\
& \boldsymbol{\theta}_{*}^{\mathrm{T}}=\left[\theta_{0 *} \theta_{1 *} \theta_{2 *} \theta_{3 *} \theta_{4 *} \ldots \theta_{5 *} \theta_{6 *}\right]
\end{aligned}
$$

where the variance of the measurement noise $\xi_{k}$ is equal to three. The first harmonic of the signal and its estimate are presented as follows

$$
\begin{aligned}
& y_{f k}=\theta_{0 *}+\theta_{1 *} \cos (k \Delta)+\theta_{2 *} \sin (k \Delta) \\
& \hat{y}_{f k}=\theta_{0 k}+\theta_{1 k} \cos (k \Delta)+\theta_{2 k} \sin (k \Delta)
\end{aligned}
$$

where adjustable parameters $\theta_{i k}, i=0,1,2$ are calculated according to two algorithms described above. Figure 1 illustrates the convergence of the estimation error $\left\|\mathbf{I}-\mathbf{D}^{-1} \mathbf{A F}_{i}\right\|, i=1,2, \ldots$ to zero for the algorithm of the matrix inversion (25) to (27). The convergence of the estimation error to zero

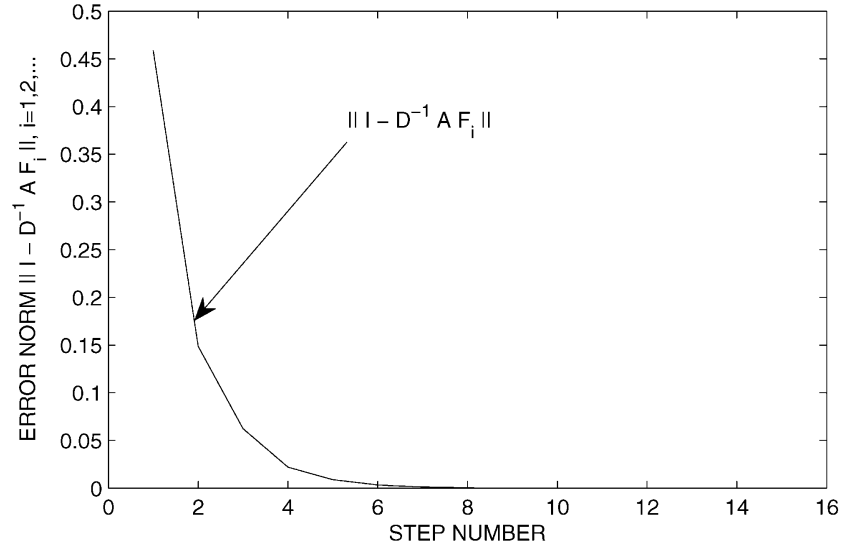

Fig. 1 The estimation error $\left\|\mathbf{I}-\mathbf{D}^{-1} \mathbf{A} \mathbf{F}_{i}\right\|, i=1,2, \ldots$ for the algorithm of the matrix inversion (25) to (27)

implies that $\mathbf{F}_{i} \mathbf{D}^{-1} \rightarrow \mathbf{A}^{-1}$ as $i \rightarrow \infty$ and the vector of adjustable parameters defined in equation (7) can then be calculated as

$$
\boldsymbol{\theta}_{k}=\left[\mathbf{F}_{i} \mathbf{D}^{-1}\right] \sum_{j=k-(w-1)}^{j=k} \boldsymbol{\varphi}_{j} y_{j}
$$

Figure 2 shows the convergence of the adjustable parameters to their true values, where the parameters are estimated via the Kaczmarz projection method. These parameters are used as initial values in algorithm (41). Both algorithms (25) to (27), (7), and (41), converge to the same vector of adjustable parameters used in TI method and defined in equation

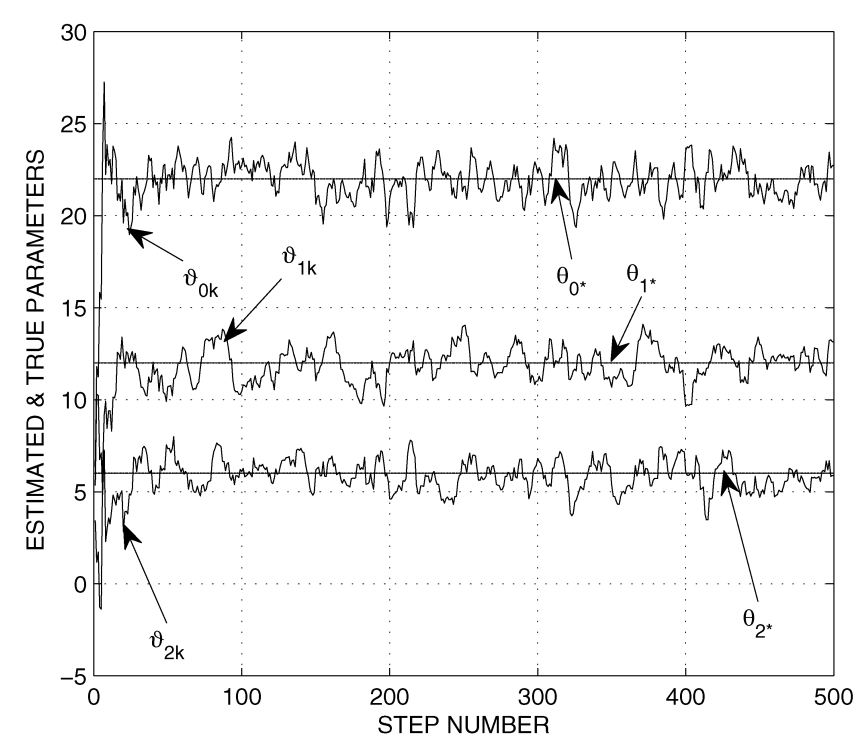

Fig. 2 Convergence of the adjustable parameters $\vartheta_{0 k}$, $\vartheta_{1 k}$, and $\vartheta_{2 k}$ calculated using the Kaczmarz projection method defined in equation (40) to their constant true values $\theta_{0 *}, \theta_{1 *}$, and $\theta_{2 *}$ defined in equation (3) respectively 


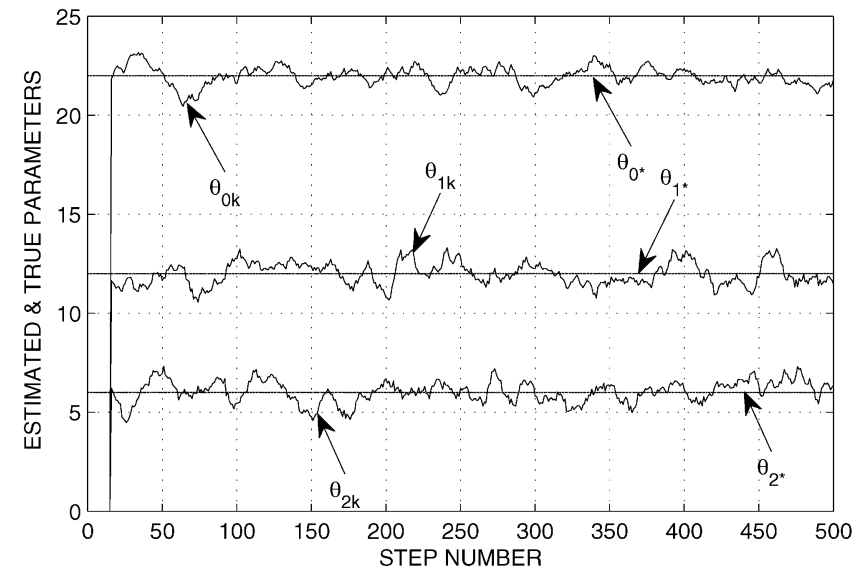

Fig. 3 Convergence of the adjustable parameters $\theta_{0 k}$, $\theta_{1 k}$, and $\theta_{2 k}$ calculated in equation (7) and used in equation (46) to their constant true values $\theta_{0 *}, \theta_{1 *}$, and $\theta_{2 *}$ defined in equation (3)

(7). The performance of the TI method is shown in Fig. 3 and Fig. 4 for window size $w=15$. Figure 3 shows the convergence of the adjustable parameters $\boldsymbol{\theta}_{k}$ defined in equation (7) to their true values $\boldsymbol{\theta}_{*}$ defined in equation (3). Figure 4 shows a final result of a successful reconstruction of the first harmonic of a periodic signal from noisy measurements.

\section{CONCLUSIONS}

The periodic nature of a wide class of machines results in oscillations in their signals. For example, the periodicity of the individual air intake of cylinders and torque production events in automotive engines results in periodicity of the intake manifold pressure and engine speed signals. This periodicity opens the opportunity to use trigonometric polynomials, in the form of equation (1) for example, for modelling machine signals. A remarkable SDD property of the information matrix created by the regressor vector that consists of trigonometric functions allows the computationally inexpensive inversion of the information matrix and establishes the property of the persistency of excitation of the oscillating signal. This in turn, opens a new class of algorithms with a reduced computational complexity for both estimation of the frequency contents of the signals and for filtering in real-time signal processing applications. Two new moving-in-time-window trigonometric interpolation algorithms of this class based on the SDD property of the information matrix established in Lemma 1, and inequality (23) were reported. The algorithms are suitable for both calculation of the frequency contents of oscillating

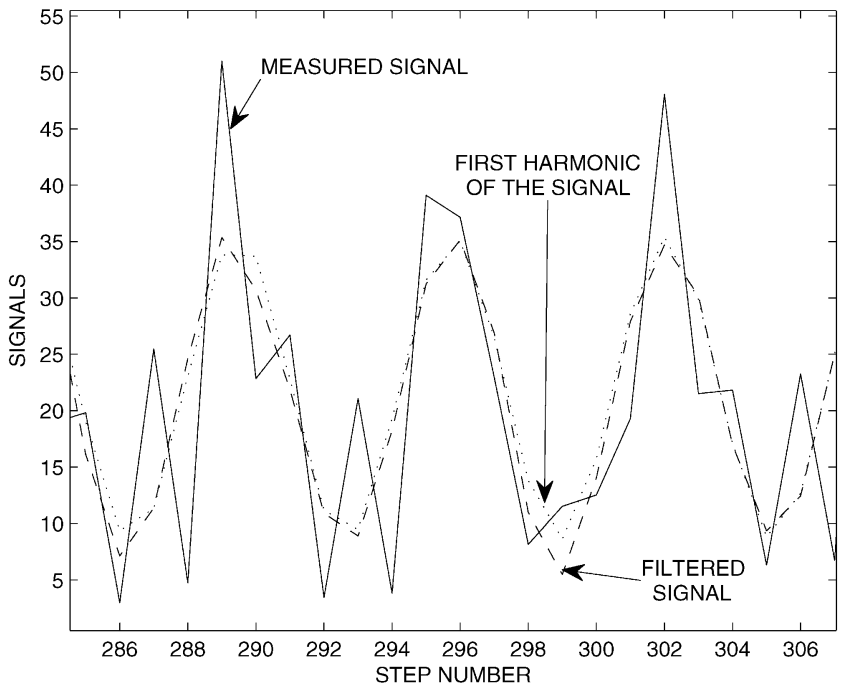

Fig. 4 Reconstruction of the first harmonic of a periodic signal. Measured signal (42) is plotted with a solid line. First harmonic (45) is plotted with a dotted line. Filtered signal (46) is plotted with a dashed line

signals and for filtering. The techniques were illustrated in the simulation example of reconstruction of the first harmonic of a periodic signal from noisy measurements.

The signal used in the simulation example is very similar to the engine speed signal in automotive engines, where high-frequency oscillations due to the crankshaft torsion together with vibrations from the road act as disturbances on the crankshaft speed signal [2]. This allows a reader to make a simple simulation set-up described in section 5 with a simulated signal which is very close to the real signal for evaluation of the results proposed in this paper.

The results described in this paper can be extended to a broader class of signals that contain both non-oscillating and oscillating components. Such signals are often met in practical applications. For example, the engine speed signal in automotive engines has both non-oscillating and oscillating parts during a transient. Non-oscillating components should be properly compensated for estimation of the frequency contents of such a signal. Some of the simple compensation techniques are reported in [2].

(c) Author 2010

\section{REFERENCES}

1 Ljung, S. and Ljung, L. Error propagation properties of recursive least-squares adaptation algorithms. Automatica, 1985, 21(2), 157-167. 
2 Stotsky, A. Automotive engines: control, estimation, statistical detection, 2009 (Springer, Berlin).

3 Horn, R. and Johnson, C. Matrix analysis, 1985 (Cambridge University Press).

4 Ljung, L. System identification: theory for the user, 1999 (Prentice Hall).

\section{APPENDICES}

\section{APPENDIX 1}

The proof of Lemma 1 is followed after Lemma 3 and Lemma 4.

\section{Lemma 3}

Consider the following sum

$$
\sum_{r=p}^{r=l} f(r)=f(p)+f(p+1)+\ldots+f(l)
$$

where $f(r)$ is a matrix function of $r$. If there exists a matrix function $\Phi(r)$ such that the following holds

$$
\Phi(r+1)-\Phi(r)=f(r)
$$

for all $r=p, \ldots, l, l>p$, then this sum can be calculated as follows

$$
\sum_{r=p}^{r=l} f(r)=\Phi(l+1)-\Phi(p)
$$

\section{Proof}

Direct substitution of $f(p)=\Phi(p+1)-\Phi(p), f(p+1)=$ $\Phi(p+2)-\Phi(p+1), \ldots, f(l)=\Phi(l+1)-\Phi(l)$ in equation (47) where all the inbetween terms vanish gives equation (49).

\section{Lemma 4}

The following relationships are valid

$$
\begin{aligned}
& \sum_{r=1}^{n} \cos (r h)=\frac{\cos [(n+1) / 2 h] \sin (n h / 2)}{\sin (h / 2)} \\
& \sum_{r=1}^{n} \sin (r h)=\frac{\sin (n h / 2) \sin [(n+1) / 2 h]}{\sin (h / 2)}
\end{aligned}
$$

Proof

Straightforward application of Lemma 3 to the calculation of the following sum $\sum_{r=1}^{n} \mathrm{e}^{\mathrm{i} h r}$ with scalar functions $f(r)=\mathrm{e}^{\mathrm{i} h r}$ and $\Phi(r)=\mathrm{e}^{\mathrm{i} h r} /\left(\mathrm{e}^{\mathrm{i} h}-1\right)$, where equation (48) is valid yields

$$
\sum_{r=1}^{n} \mathrm{e}^{\mathrm{i} h r}=\frac{\mathrm{e}^{\mathrm{i} h}\left(1-e^{\mathrm{i} h n}\right)}{\left(1-\mathrm{e}^{\mathrm{i} h}\right)}
$$

where $\mathrm{i}^{2}=-1$, and $\mathrm{e}^{\mathrm{i} h} \neq 1$. Taking into account the following relations $1-\mathrm{e}^{\mathrm{i} h}=-2 \mathrm{i} \sin (h / 2) \mathrm{e}^{\mathrm{i} h / 2}, 1-\mathrm{e}^{\mathrm{i} h n}$ $=-2 \mathrm{i} \sin (n h / 2) \mathrm{e}^{\mathrm{i} n h / 2}$, equation (52) can be written as

$$
\sum_{r=1}^{n} \mathrm{e}^{\mathrm{i} h r}=\mathrm{e}^{\mathrm{i} h(n+1) / 2} \frac{\sin (n h / 2)}{\sin (h / 2)}
$$

Equations (50) and (51) follow from equation (53) which can be written as

$$
\begin{aligned}
\sum_{r=1}^{n} & \cos (r h)+i \sum_{r=1}^{n} \sin (r h) \\
= & \frac{\cos [(n+1) / 2 h] \sin (n h / 2)}{\sin (h / 2)} \\
& +i \frac{\sin (n h / 2) \sin [(n+1) / 2 h]}{\sin (h / 2)}
\end{aligned}
$$

when equating real and imaginary parts.

\section{Remark 4}

Equations (50) and (51) can also be calculated via a direct application of Lemma 3 with

$$
\begin{aligned}
& \Phi(r)=\frac{\cos (r h / 2) \sin [(r-1) / 2 h]}{\sin (h / 2)} \text { and } \\
& f(r)=\cos (r h)
\end{aligned}
$$

for equation (50), and with

$$
\begin{aligned}
& \Phi(r)=\frac{\sin (r h / 2) \sin [(r-1) / 2 h]}{\sin (h / 2)} \quad \text { and } \\
& f(r)=\sin (r h)
\end{aligned}
$$

for equation (51). Straightforward calculations show that equation (48) is valid in both cases and equations (50) and (51) follow immediately from equation (49) with $p=1$ and $l=n$.

\section{Proof of Lemma 1}

The proof is based on explicit calculation of the elements of the matrix A using Lemma 4

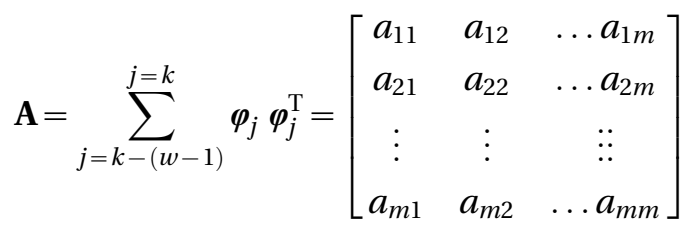


where

$$
\begin{aligned}
& a_{11}=w, \quad a_{12}=\sum_{j=k-(w-1)}^{j=k} \cos (j \Delta) \\
& a_{13}=\sum_{j=k-(w-1)}^{j=k} \sin (j \Delta), \quad a_{1 m}=\sum_{j=k-(w-1)}^{j=k} \sin (j n \Delta) \\
& a_{21}=a_{12}, \quad a_{22}=\sum_{j=k-(w-1)}^{j=k} \cos ^{2}(j \Delta) \\
& a_{2 m}=\sum_{j=k-(w-1)}^{j=k} \cos (j \Delta) \sin (j n \Delta), \quad a_{m 1}=a_{1 m} \\
& a_{m 2}=a_{2 m}, \quad a_{m m}=\sum_{j=k-(w-1)}^{j=k} \sin ^{2}(j n \Delta)
\end{aligned}
$$

where $m=(2 n+1)$.

First, the following sums are calculated

$$
\begin{aligned}
& \sum_{j=1}^{k} \cos (j q \Delta)=\frac{\cos [(k+1) / 2 q \Delta] \sin (k q \Delta / 2)}{\sin (q \Delta / 2)} \\
& \sum_{j=1}^{k-w} \cos (j q \Delta) \\
& \quad=\frac{\cos [(k-w+1) / 2 q \Delta] \sin [(k-w / 2) q \Delta]}{\sin (q \Delta / 2)}
\end{aligned}
$$

where $q=1, \ldots, n$, and $n$ is the number of the frequencies. The sums (54) and (55) follow from equation (50) with $h=q \Delta, n=k$ for equation (54) and $n=k-w$ for equation (55).

The sum of $\cos (j q \Delta), j=k-(w-1), \ldots, k$ in the window of a size $w$ is calculated as follows

$$
\begin{aligned}
& \sum_{j=k-(w-1)}^{j=k} \cos (j q \Delta)=\sum_{j=1}^{k} \cos (j q \Delta)-\sum_{j=1}^{k-w} \cos (j q \Delta) \\
& =\frac{\sin (w q \Delta / 2) \cos [(2 k+1-w) / 2 q \Delta]}{\sin (q \Delta / 2)}
\end{aligned}
$$

The sum of $\sin (j q \Delta), j=k-(w-1), \ldots, k$ in the window of a size $w$ can be written as follows

$$
\begin{aligned}
& \sum_{j=k-(w-1)}^{j=k} \sin (j q \Delta) \\
& =\frac{\sin (w q \Delta / 2) \sin [(2 k+1-w) / 2 q \Delta]}{\sin (q \Delta / 2)}
\end{aligned}
$$

Using similar arguments all non-diagonal elements of the matrix A are evaluated. Evaluation of the diagonal elements yields

$$
\begin{aligned}
& \sum_{j=k-(w-1)}^{j=k} \sin ^{2}(j q \Delta) \\
& =\frac{w}{2}-\frac{\sin (w q \Delta) \cos ((2 k+1-w) q \Delta)}{2 \sin (q \Delta)} \\
& \sum_{j=k-(w-1)}^{j=k} \cos ^{2}(j q \Delta) \\
= & \underbrace{\frac{w}{2}}_{\text {average part }}+\underbrace{\frac{\sin (w q \Delta) \cos ((2 k+1-w) q \Delta)}{2 \sin (q \Delta)}}_{\text {periodic part }}
\end{aligned}
$$

Notice that all the diagonal elements $a_{i i}, i=2, \ldots$, $m$ of the matrix $\mathbf{A}$ which are calculated according to equations (58) and (59) have an average part which depends on the window size $w$, namely $w / 2$, and a periodic part. Non-diagonal elements $a_{i j}, i \neq j$ of the matrix A have periodic parts only. All diagonal elements are increasing if the size of the window $w$ increases (see equations (58) and (59)). Non-diagonal elements of the matrix remain bounded if the size of the window $w$ increases. There exists therefore a window size $w_{*}$ such that for all $w>w_{*}$ the matrix $\mathbf{A}$ is an SDD matrix, where $w_{*}$ is defined as

$$
\begin{aligned}
w_{*}=\operatorname{round}\left\{\max :\left[\sum_{j=2}^{2 n+1}\left|a_{1 j}\right|, \quad 2\left(\sum_{j=1, j \neq i}^{2 n+1}\left|a_{i j}\right|\right.\right.\right. \\
\left.\left.\left.+\left|a_{i i p}\right|\right), \quad i=2, \ldots,(2 n+1)\right]\right\}
\end{aligned}
$$

where round is understood as rounding to the largest integer and $a_{i i p}$ is a periodic part of the diagonal elements, $i=2, \ldots,(2 n+1)$.

This symmetric SDD matrix A has positive eigenvalues only and hence equation (12) holds for $\alpha=\lambda_{\min }(\mathbf{A})$, and $\beta=\lambda_{\max }(\mathbf{A})$, where $\lambda_{\min }(\mathbf{A}), \lambda_{\max }(\mathbf{A})$ are the minimal and maximal eigenvalues of the matrix $\mathbf{A}[\mathbf{2}, \mathbf{3}]$. Notice that every eigenvalue $\lambda$ of $\mathbf{A}$ is located within at least one of the Gershgorin discs 


$$
\left|\lambda-a_{i i}\right| \leqslant \sum_{j=1, j \neq i}^{2 n+1}\left|a_{i j}\right|, \quad \text { for } i=1, \ldots,(2 n+1)
$$

which shows that the eigenvalues are close to the diagonal elements $a_{i i}$ of the matrix $\mathbf{A}$ if $a_{i i} \gg \sum_{j=1, j \neq i}^{2 n+1}\left|a_{i j}\right|$. The following bounds are true for minimal and maximal eigenvalues of the matrix $\mathbf{A}$

$$
\begin{aligned}
& \lambda_{\min } \geqslant \min \left\{a_{i i}-\sum_{j=1, j \neq i}^{2 n+1}\left|a_{i j}\right|\right\}>0 \\
& \lambda_{\max } \leqslant \max \left\{a_{i i}+\sum_{j=1, j \neq i}^{2 n+1}\left|a_{i j}\right|\right\}
\end{aligned}
$$

where $i=1, \ldots,(2 n+1)$.

Notice that this proof is valid for the case where regressor (2) only contains distinct frequencies. Sums of squares of trigonometric functions (that contain an average part) appear as non-diagonal elements in the information matrix if regressor (2) has two or more identical frequencies. Diagonal dominance of the information matrix is destroyed in this case since non-diagonal elements also increase when the window size increases.

\section{APPENDIX 2}

\section{Proof of Lemma 2}

Consider the following sum $\sum_{i=0}^{N} \mathbf{B}^{i}$ where $\mathbf{B}$ is an $n \times n$ matrix. Application of Lemma 3 with $f(i)=\mathbf{B}^{i}$, $r=i$, and $\Phi(i)=\mathbf{B}^{i}(\mathbf{B}-\mathbf{I})^{-1}$ where equation (48) is valid yields

$$
\sum_{i=0}^{N} \mathbf{B}^{i}=\left(\mathbf{B}^{N+1}-\mathbf{I}\right)(\mathbf{B}-\mathbf{I})^{-1}
$$

Choosing $\mathbf{B}$ as $\mathbf{B}=(\mathbf{I}-\mathbf{C})$ the sum of equation (62) is

$$
\sum_{i=0}^{N}(\mathbf{I}-\mathbf{C})^{i}=\left((\mathbf{I}-\mathbf{C})^{N+1}-\mathbf{I}\right)(-\mathbf{C})^{-1}
$$

Taking into account that $\left\|(\mathbf{I}-\mathbf{C})^{N+1}\right\| \leqslant$ $\|(\mathbf{I}-\mathbf{C})\|^{N+1} \leqslant \kappa^{N+1} \rightarrow 0$ as $N \rightarrow \infty$ equation (24) follows from equation (63).

\section{APPENDIX 3}

\subsection{Evaluation of the convergence rate of algorithm (25) to (27)}

First, algorithm (25) to (27) is listed here for convenience

$$
\begin{aligned}
& \mathbf{F}_{1}=\mathbf{I}, \quad \mathbf{G}_{1}=\left(\mathbf{I}-\mathbf{D}^{-1} \mathbf{A}\right) \\
& \mathbf{G}_{i}=\mathbf{G}_{i-1} \mathbf{G}_{1} \\
& \mathbf{F}_{i}=\mathbf{F}_{i-1}+\mathbf{G}_{i-1}
\end{aligned}
$$

where $i=2,3, \ldots$.

The following difference

$$
\mathbf{F}_{i} \mathbf{D}^{-1}-\mathbf{A}^{-1}=\left(\mathbf{F}_{i} \mathbf{D}^{-1} \mathbf{A}-\mathbf{I}\right) \mathbf{A}^{-1}
$$

is used for evaluation of the convergence rate of algorithm (64) to (66) that provides $\mathbf{F}_{i} \mathbf{D}^{-1} \rightarrow \mathbf{A}^{-1}$ as $i \rightarrow \infty$, where $\mathbf{F}_{i}$ is defined in equation (66). The following matrix $\mathbf{R}_{i}=\mathbf{F}_{i} \mathbf{D}^{-1} \mathbf{A}-\mathbf{I}$, for $i=1,2,3, \ldots$ is considered first. Straightforward calculations show that $\quad \mathbf{R}_{1}=\mathbf{D}^{-1} \mathbf{A}-\mathbf{I}, \quad \mathbf{R}_{2}=-\mathbf{R}_{1}^{2}, \quad \mathbf{R}_{3}=\mathbf{R}_{1}^{3}, \quad \ldots, \quad \mathbf{R}_{i}=$ $(-1)^{i-1} \mathbf{R}_{1}^{i}, \quad i=1,2,3, \ldots$ and $\left\|\mathbf{R}_{i}\right\| \leqslant\left\|\mathbf{R}_{1}\right\|^{i} \leqslant \kappa^{i}$ since equation (23) holds. Evaluation of $\mathbf{A}^{-1}$ yields

$$
\begin{gathered}
\mathbf{A}^{-1}=\left(\mathbf{A}^{-1} \mathbf{D}\right) \mathbf{D}^{-1} \stackrel{(29)}{\rightarrow}\left[\sum_{i=0}^{N}\left(\mathbf{I}-\mathbf{D}^{-1} \mathbf{A}\right)^{i}\right] \mathbf{D}^{-1} \\
\quad \text { as } \quad N \rightarrow \infty
\end{gathered}
$$

hence

$$
\begin{aligned}
\mathbf{A}^{-1}= & {\left[\mathbf{I}+\left(\mathbf{I}-\mathbf{D}^{-1} \mathbf{A}\right)+\left(\mathbf{I}-\mathbf{D}^{-1} \mathbf{A}\right)^{2}\right.} \\
& \left.+\left(\mathbf{I}-\mathbf{D}^{-1} \mathbf{A}\right)^{3}+\ldots\right] \mathbf{D}^{-1}
\end{aligned}
$$

The upper bound of the norm of the matrix $\mathbf{A}^{-1}$ follows from equation (68) by taking into account equation (23) 


$$
\begin{aligned}
\left\|\mathbf{A}^{-1}\right\| & \leqslant\left(1+\kappa+\kappa^{2}+\kappa^{3}+\ldots\right)\left\|\mathbf{D}^{-1}\right\| \\
& =\frac{1}{1-\kappa}\left\|\mathbf{D}^{-1}\right\|
\end{aligned}
$$

where $\|\mathbf{I}\|=1$ and the sum of geometric progression written in the brackets is calculated using Lemma 3 with $\Phi(r)=\kappa^{r} /(\kappa-1)$ and $f(r)=\kappa^{r}$.

Finally, the convergence rate for algorithm (64) to (66) is evaluated as follows

$$
\begin{aligned}
\left\|\mathbf{F}_{i} \mathbf{D}^{-1}-\mathbf{A}^{-1}\right\| & \leqslant\left\|\left(\mathbf{F}_{i} \mathbf{D}^{-1} \mathbf{A}-\mathbf{I}\right)\right\|\left\|\mathbf{A}^{-1}\right\| \\
& =\left\|\mathbf{R}_{i}\right\|\left\|\mathbf{A}^{-1}\right\| \leqslant \frac{\kappa^{i}}{1-\kappa}\left\|\mathbf{D}^{-1}\right\|
\end{aligned}
$$

\subsection{Evaluation of the convergence rate of algorithm (30) to (34)}

First, algorithm (30) to (34) is listed here for convenience

$$
\begin{aligned}
& \mathbf{G}_{i}=\mathbf{G}_{i-1}+\mathbf{F}_{i-1} \mathbf{G}_{i-1} \\
& \mathbf{F}_{i}=\mathbf{I}-\mathbf{G}_{i} \mathbf{A} \\
& \mathbf{F}_{0}=\mathbf{I}-\mathbf{G}_{0} \mathbf{A} \\
& \mathbf{G}_{0}=\mathbf{D}^{-1}
\end{aligned}
$$$$
\left\|\mathbf{F}_{0}\right\| \stackrel{(23)}{\leqslant} \kappa<1
$$

where $i=1,2,3, \ldots$

Notice that the following relations are valid

$$
\begin{aligned}
\mathbf{F}_{1} & =\mathbf{I}-\mathbf{G}_{1} \mathbf{A}=\mathbf{I}-\left(\mathbf{G}_{0}+\mathbf{F}_{0} \mathbf{G}_{0}\right) \mathbf{A} \\
& =\mathbf{I}-\left(\mathbf{I}+\mathbf{F}_{0}\right) \mathbf{G}_{0} \mathbf{A}=\mathbf{I}-\left(\mathbf{I}+\mathbf{F}_{0}\right)\left(\mathbf{I}-\mathbf{F}_{0}\right) \\
& =\mathbf{I}-\left(\mathbf{I}-\mathbf{F}_{0}^{2}\right)=\mathbf{F}_{0}^{2} \\
\mathbf{F}_{2} & =\mathbf{F}_{1}^{2}=\mathbf{F}_{0}^{4} \\
\mathbf{F}_{i} & =\mathbf{F}_{0}^{2^{i}}
\end{aligned}
$$

$$
\left\|\mathbf{F}_{i}\right\| \leqslant\left\|\mathbf{F}_{0}^{2^{i}}\right\| \leqslant \kappa^{2^{i}}
$$

The convergence rate of the algorithm can be evaluated as follows

$$
\begin{aligned}
\left\|\mathbf{A}^{-1}-\mathbf{G}_{i}\right\| & \leqslant\left\|\mathbf{I}-\mathbf{G}_{i} \mathbf{A}\right\|\left\|\mathbf{A}^{-1}\right\| \\
& =\left\|\mathbf{F}_{i}\right\|\left\|\mathbf{A}^{-1}\right\| \leqslant\left\|\mathbf{A}^{-1}\right\| \kappa^{2^{k}}
\end{aligned}
$$

Taking into account the relationship which follows from equations (62) and (75)

$$
\begin{gathered}
\sum_{i=0}^{N} \mathbf{F}_{0}^{i}=\left(\mathbf{F}_{0}^{N+1}-\mathbf{I}\right)\left(\mathbf{F}_{0}-\mathbf{I}\right)^{-1} \rightarrow\left(\mathbf{I}-\mathbf{F}_{0}\right)^{-1} \\
\text { as } \quad N \rightarrow \infty
\end{gathered}
$$

the matrix $\mathbf{A}^{-1}$ is evaluated using equation (73) as follows

$$
\begin{aligned}
\mathbf{A}^{-1} & =\left(\mathbf{I}-\mathbf{F}_{0}\right)^{-1} \mathbf{G}_{0}=\left[\sum_{i=0}^{N} \mathbf{F}_{0}^{i}\right] \mathbf{G}_{0} \\
& =\left(\mathbf{I}+\mathbf{F}_{0}+\mathbf{F}_{0}^{2}+\ldots\right) \mathbf{G}_{0}
\end{aligned}
$$

Therefore, the following upper bound of the norm of the matrix $\mathbf{A}^{-1}$ is valid

$$
\begin{aligned}
\left\|\mathbf{A}^{-1}\right\| & =\left\|\left(\mathbf{I}-\mathbf{F}_{0}\right)^{-1}\right\|\left\|\mathbf{G}_{0}\right\| \\
& \leqslant\left(\|\mathbf{I}\|+\kappa+\kappa^{2}+\ldots\right)\left\|\mathbf{G}_{0}\right\| \\
& \leqslant\left(1+\kappa+\kappa^{2}+\ldots\right)\left\|\mathbf{G}_{0}\right\| \\
& =\frac{1}{1-\kappa}\left\|\mathbf{G}_{0}\right\|
\end{aligned}
$$

since

$$
\sum_{i=0}^{N} \kappa^{i}=\frac{1-\kappa^{N+1}}{1-\kappa} \rightarrow \frac{1}{1-\kappa} \text { as } N \rightarrow \infty
$$

with a valid inequality equation (75).

Finally, the following bound is valid for the estimation error

$$
\left\|\mathbf{A}^{-1}-\mathbf{G}_{i}\right\| \leqslant \frac{\left\|\mathbf{D}^{-1}\right\|}{1-\kappa} \kappa^{2^{i}}
$$

A comparison of two upper bounds (70) and (81) shows that algorithm (30) to (34) converges faster than algorithm (25) to (27). Indeed, the convergence 
rate, as can be seen from equations (77) and (70), for algorithm (30) to (34) is determined by $\mathbf{F}_{i}=\mathbf{F}_{0}^{2^{i}}$, $\mathbf{F}_{0}=\mathbf{I}-\mathbf{D}^{-1} \mathbf{A}$ and by $\mathbf{R}_{i}=(-1)^{i-1} \mathbf{R}_{1}^{i}, \mathbf{R}_{1}=\mathbf{D}^{-1} \mathbf{A}-\mathbf{I}$, $i=1,2,3, \ldots$ for algorithm (25) to (27). A comparison of these two sequences of matrices shows that algorithm (25) to (27) has a linear convergence rate whereas algorithm (30) to (34) has a quadratic convergence rate. Faster convergence of algorithm (30) to (34) can also be confirmed by simulations. 